\title{
ПРОГРАММНЫЙ МОДУЛЬ ПОДДЕРЖКИ ПРИНЯТИЯ РЕШЕНИЙ ПРИ ФОРМИРОВАНИИ ИНВЕСТИЦИОННОГО ПОРТФЕЛЯ
}

\section{Тюльпинова Н.В.}

В современных условиях проблема повышения обоснованности инвестиций имеет исключительно важное значение для развития экономики предприятий, поскольку ошибочные инвестиционные решения относительно вложения средств в недостаточно эффективные проекты могут повлечь за собой существенные убытки вплоть до разорения предприятий. Невозможно оценить рентабельность того или иного инвестичионного портфеля, основываясь лишь на личном опыте и интуиции, - необходимо расчетное моделирование инвестиционного процесса, требующее значительного объема вычислений. Однако, несмотря на то, что методология такого моделирования на концептуальном уровне опубликована и изложена достаточно подробно, отсутствие конкретных алгоритмических рекомендаций относительно программной реализации её вычислительной схемы препятствуют широкому её внедрению в инвестиционную практику. В этой связи актуальной является разработка специилизированного алгоритмического и программного обеспечения, предоставляющего инвестору гибкий инструмент принятия обоснованных решений.

Цель - повышение обоснованности принятия решений при формировании портфеля инвестиционных проектов.

Метод или методология проведения работы: динамическое программирование.

Результаты: программно-алгоритмический инструментарий поддержки принятия решений при формировании инвестищионного портфеля. 
Область применения результатов: менеджмент (инвестиционный, инновационныгй, финансовый, стратегический, антикризисный, риск-менеджмент).

Ключевые слова: инвестиционный портфель; динамическое программирование; оптимизация.

\section{THE SOFTWARE MODULE OF SUPPORT DECISION-MAKING FOR INVESTMENT PORTFOLIO}

\section{Tyulpinova N.V.}

In the modern conditions, the problem of increasing the validity of investments is extremely important for the development of the enterprise economy, since in the case of erroneous investment decisions regarding investment in insufficiently effective projects can lead to significant losses up to the ruin of enterprises. It is impossible to assess prove-in performance of an investment portfolio, based only on personal experience and intuitionit is necessary numerical simulation of the investment process, requiring large-scale computations. However, despite the fact that the methodology of such modeling at the conceptual level has been published and described in details, the lack of specific algorithmic guiding suggestions regarding the software-programmable computing prevents its all-around implementation in investment practice. In this regard, it is important to develop application-specific algorithms and software that provides investors with a flexible tool for making valid decisions.

Purpose - improving the validity of decision-making for investment portfolio.

Method or methodology of the work: dynamic programming.

Results: software and algorithms to support decision-making for investment portfolio.

Practical implications: management (investment, innovative, financial, strategic, crisis, risk).

Keywords: investment portfolio; dynamic programming; optimization. 
В процессе управления финансовыми активами постоянно возникает необходимость определения такого набора инвестиционных проектов, реализация которого позволит достичь максимального эффекта в условиях существующих ограничений. Основная задача при этом заключается в нахождении оптимального распределения инвестиций по объектам вложения, которое обеспечит максимально возможный суммарный доход при условии, что суммарные затраты на реализацию проектов портфеля не превысят имеющихся средств.

Поставленная задача может быть решена как методом полного перебора всех возможных вариантов и выбора среди них наилучшего, так и методом динамического программирования. Сопоставление этих методов с точки зрения вычислительной сложности, эффективности и быстродействия однозначно говорит о существенном преимуществе второго метода, так как главной вычислительной особенностью динамического программирования является декомпозиция исходной многомерной задачи на ряд взаимосвязанных одномерных задач. Однако, несмотря на то, что второй метод на концептуальном уровне опубликован и изложен достаточно подробно [1-15], описание его специфики, в большинстве случаев, включает лишь общую формульно-словесную трактовку, которая: 1) не имеет достаточно строгой формализации для последующей её реализации на ЭВМ; 2) не раскрывает сущность конкретной конечной последовательности однозначных предписаний, исполнение которой позволит с помощью конечного числа шагов получить решение задачи на ЭВМ, однозначно определяемое исходными данными; 3) не содержит наглядных, развернутых, детализированных алгоритмов, что допускает как неоднозначность толкования отдельных положений рассматриваемого метода, так и неопределенность относительно наиболее приемлемых в каждом конкретном случае структур данных и способов их обработки. Отсутствие конкретных алгоритмических рекомендаций относительно практической реализации вычислительной схемы метода динамического программирования существенно усложняет разработку реализующего его прикладного 
программного обеспечения, при том, что без автоматизации практическое применение данного метода весьма затруднительно, так как его алгоритм очень трудоемок для ручных расчетов по причине необходимости выполнения большого объема повторяющихся действий, основанных на решении функциональных уравнений. Всё это в совокупности препятствует широкому внедрению данного метода в инвестиционную практику.

В этой связи представленные ниже результаты исследований прикладной программный модуль и его алгоритмическая структура - являются актуальными и представляют интерес как с научной, так и с практической точки зрения, а именно: разработанный алгоритм легко может быть закодирован на любом современном языке программирования любым специалистом, обладающим общими знаниями в области программирования, а разработанный программный модуль позволит инвестору оперативно (за доли секунды) формировать оптимальный инвестиционный портфель, который принесёт максимальную прибыль.

В основу разработанного алгоритмического и программного обеспечения положены основные концепции метода динамического программирования.

Структура алгоритмического ядра формирования оптимального инвестиционного портфеля представлена на рис. 1. Ввод исходных данных реализуется в два этапа:

1) в блоке 1 организован первый этап ввода исходных данных, которыми являются: $\mathrm{N}$ - общее число инвестиционных проектов; C - общий объем капиталовложений; $\mathrm{P}$ - кратность инвестиций; в блоке 2 происходит перевод исходных данных в безразмерную форму;

2) в блоках 3-6 посредством двойного цикла организован второй этап ввода исходных данных, которыми является двумерный массив (таблица) r[i,j] (i, j - переменные циклов), характеризующий распределение прибыли от вложений в проекты: размерности внешнего цикла $\mathrm{N}$ (блок 3 ) и внутреннего цикла $\mathrm{m}$ (блок 4) определены в блоках 1-2; в блоке 5 реализован собственно ввод числовых значений; в блоке 6 происходит их перевод в безразмерную форму. 


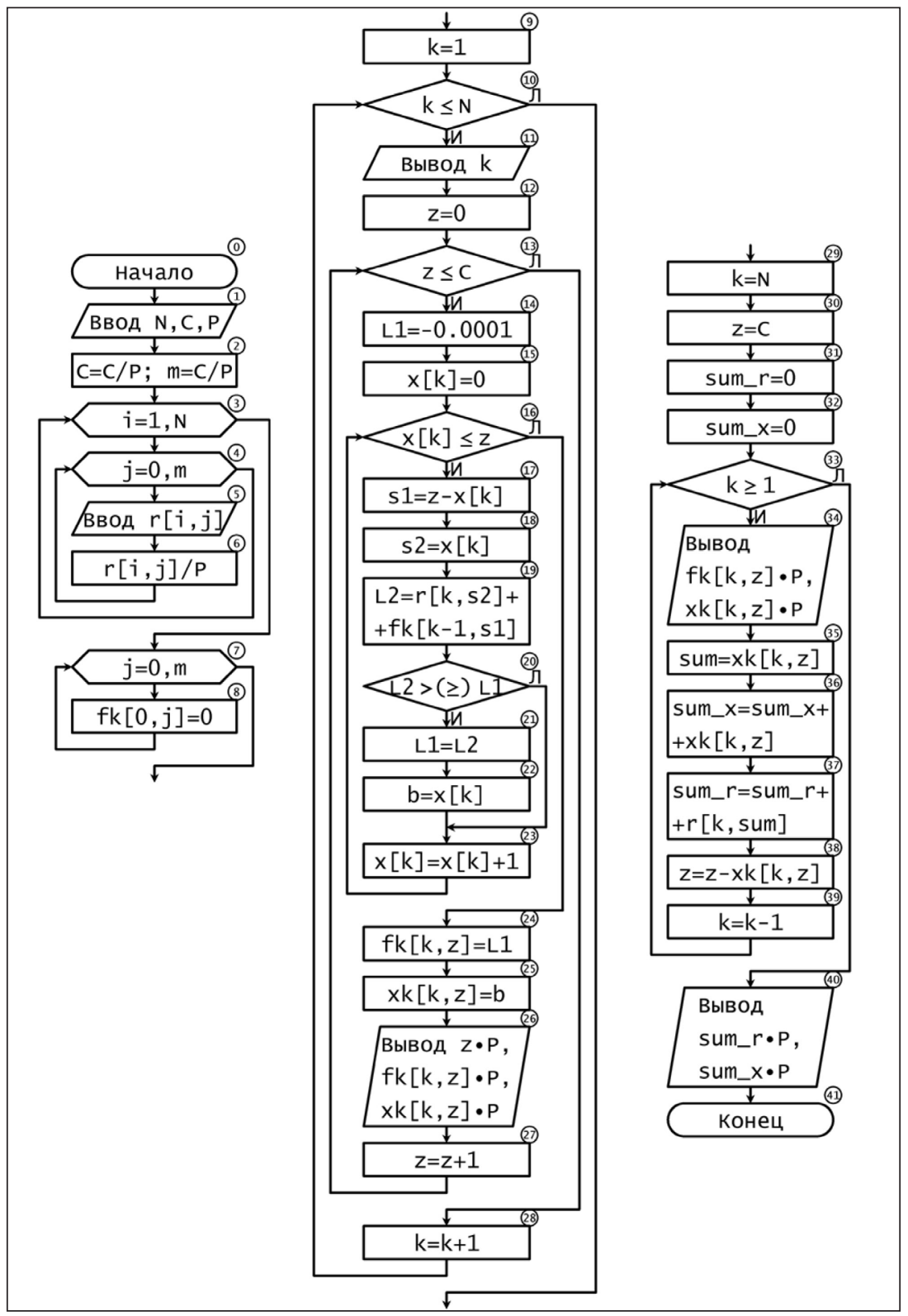

Рис. 1. Алгоритм формирования оптимального инвестиционного портфеля 
После ввода исходных данных осуществляется собственно расчет оптимального инвестиционного портфеля, который осуществляется в три этапа:

1) подготовительный этап (блоки 7-8);

2) прямой ход алгоритма динамического программирования (блоки 9-28);

3) обратный ход алгоритма динамического программирования (блоки 29-40).

Ключевыми переменными расчета являются: $\mathrm{k}$ - номер инвестиционного проекта; $\mathrm{z}$ - инвестиции; $\mathrm{fk}$ - прибыль от k фирм при инвестициях z; xk - инвестиции в каждую из фирм; sum_r - суммарная прибыль; sum_x - суммарные инвестиции.

В процессе подготовительного этапа в цикле блока 7 размерностью т происходит обнуление прибыли (блок 8).

Прямой ход алгоритма реализован в двойном цикле: внешний цикл перебора по проектам - блок 10 (начальное значение для данного цикла задается в блоке 9, переход к следующей итерации - в блоке 28) и внутренний цикл поиска оптимального вложения в конкретный проект - блок 13 (начальное значение для данного цикла задается в блоке 12, переход к следующей итерации - в блоке 27). Расчет реализуется в блоках 14-25: здесь реализован традиционный в программировании алгоритм поиска максимума (блоки 14, 20 :

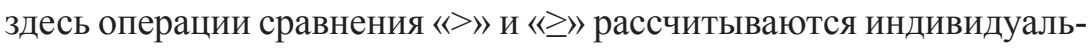
но, поскольку не всегда приводят к одинаковым ответам), а также вспомогательный цикл (блок 16, начальное значение для которого задается в блоке 15, а переход к следующей итерации - в блоке 23). Посредством блоков 11 и 26 ведется протокол расчета, содержащий таблицы результатов прямого хода алгоритма в натуральной (не безразмерной) форме для каждого проекта.

Обратный ход алгоритма реализован в цикле блока 33 (начальные значения для данного цикла задаются в блоках 29-32, переход к следующей итерации - в блоке 39). Расчет осуществляется в блоках 35-39 указанного цикла, а вывод результатов - оптимального распределения капиталовложений в натуральной форме - в блоке 34. В блоке 40 реализован вывод в натуральной форме максималь- 
ной суммарной прибыли, а также суммарных затрат (для контроля правильности вычислений).

Пользовательский интерфейс программного обеспечения, разработанного на базе описанного алгоритмического ядра, представлен на рис. 2:

1) окно ввода исходных данных (рис. 2a);

2) окно вывода результатов в табличной и графической форме (рис. 2б);

3) окно детализации произведенных расчетов (рис. 2в).

В целях контроля точности работы разработанного программного обеспечения на рис. 3 приведен верификационный пример с подробным протоколом расчета для исходных данных, указанных на рис. $2 \mathrm{a}$.

Протокол расчёта включает четыре блока:

1) блок ввода исходных данных (рис. 3a);

2) блок графической интерпретации исходных данных (рис. 3б);

3) вычислительный блок для установленной операции сравнения «>» в блоке 20 алгоритмического ядра (рис. 3б);

4) вычислительный блок для установленной операции сравнения «邓» в блоке 20 алгоритмического ядра (рис. 3в).

На рис. 2 представлен результат работы программного модуля для случая, когда операции сравнения «>» и «» приводят к неодинаковым (несовпадающим) ответам. В качестве альтернативы на рис. 4 представлен результат для случая с одинаковыми (совпадающими) ответами.

Разработанный программно-алгоритмический инструментарий позволяет оперативно, в автоматическом режиме вырабатывать надежные и обоснованные управленческие решения относительно формирования оптимального инвестиционного портфеля, обеспечивающего максимальную прибыль в заданных (имеющихся) условиях. Детальное описание вычислительного алгоритма делает его доступным для кодирования на любом современном языке программирования с целью разработки соответствующих приложений. Пользовательский интерфейс прост в освоении, не требует от инвестора специализированной подготовки и позволяет приступить к работе сразу после инсталляции. 


\begin{tabular}{|c|c|c|c|c|}
\hline \multicolumn{5}{|c|}{ OPIMAL INUESIMENT: INPUT } \\
\hline 4 & \multirow{2}{*}{\multicolumn{4}{|c|}{$\begin{array}{l}\text { общее число инвестиционных проектов } \\
\text { общий объём капиталовложений, млн. руб }\end{array}$}} \\
\hline 700 o & & & & \\
\hline $100 \quad \mathrm{~K}$ & \multicolumn{4}{|c|}{ кратность инвестиций, мАн. руб. ВВОД } \\
\hline \multicolumn{5}{|c|}{ Распределение прибыли от вложений в проекты } \\
\hline ИНВЕСТ & СТ ПР-№1 & ППР-№2 & ПР-№3 & ПР-№4 \\
\hline 0 & 0 & 0 & 0 & 0 \\
\hline 100 & 37 & 47 & 48 & 85 \\
\hline 200 & 64 & 70 & 75 & 90 \\
\hline 300 & 87 & 80 & 98 & 111 \\
\hline 400 & 105 & 86 & 120 & 118 \\
\hline 500 & 120 & 91 & 132 & 124 \\
\hline 600 & 134 & 94 & 144 & 129 \\
\hline 700 & 145 & 98 & 156 & 132 \\
\hline \multicolumn{5}{|c|}{ РАСЧЁТ ОПТИМААЬНОГО ИНВЕСТИРОВАНИЯ } \\
\hline
\end{tabular}

a)

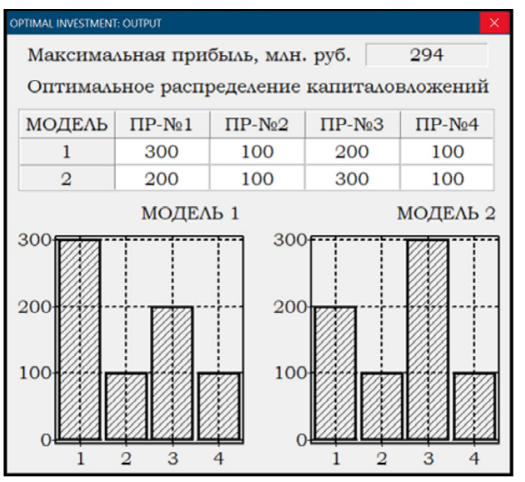

б)

\begin{tabular}{|c|c|c|c|c|c|c|c|c|}
\hline \multicolumn{9}{|c|}{ OPIIMAL INYESIMENT: DEIAIL } \\
\hline \multicolumn{9}{|c|}{ МОДЕ АЬ 1} \\
\hline z & $f 1(Z)$ & $\mathrm{x} 1(\mathrm{Z})$ & $f 2(Z)$ & $\mathrm{x} 2(\mathrm{Z})$ & $\mathrm{f} 3(\mathrm{Z})$ & $\mathrm{x} 3(\mathrm{Z})$ & $\mathrm{f} 4(\mathrm{Z})$ & $x^{4}(Z)$ \\
\hline 0 & 0 & 0 & 0 & 0 & 0 & 0 & & \\
\hline 100 & 37 & 100 & 47 & 100 & 48 & 100 & & \\
\hline 200 & 64 & 200 & 84 & 100 & 95 & 100 & & \\
\hline 300 & 87 & 300 & 111 & 100 & 132 & 100 & & \\
\hline 400 & 105 & 400 & 134 & 100 & 159 & 100 & & \\
\hline 500 & 120 & 500 & 157 & 200 & 186 & 200 & & \\
\hline 600 & 134 & 600 & 175 & 200 & 209 & 200 & & \\
\hline 700 & 145 & 700 & 190 & 200 & 232 & 200 & 294 & 100 \\
\hline \multicolumn{9}{|c|}{ МОДЕАЬ 2} \\
\hline Z & $\mathrm{f} 1(\mathrm{Z})$ & $\mathrm{x} 1(\mathrm{Z})$ & $\mathrm{f} 2(\mathrm{Z})$ & $\mathrm{x} 2(\mathrm{Z})$ & $\mathrm{f} 3(\mathrm{Z})$ & $x 3(Z)$ & $\mathrm{f} 4(\mathrm{Z})$ & $x 4(Z)$ \\
\hline 0 & 0 & 0 & 0 & 0 & 0 & 0 & & \\
\hline 100 & 37 & 100 & 47 & 100 & 48 & 100 & & \\
\hline 200 & 64 & 200 & 84 & 100 & 95 & 100 & & \\
\hline 300 & 87 & 300 & 111 & 100 & 132 & 100 & & \\
\hline 400 & 105 & 400 & 134 & 200 & 159 & 200 & & \\
\hline 500 & 120 & 500 & 157 & 200 & 186 & 200 & & \\
\hline 600 & 134 & 600 & 175 & 200 & 209 & 300 & & \\
\hline 700 & 145 & 700 & 190 & 200 & 232 & 300 & 294 & 100 \\
\hline
\end{tabular}

в)

Рис. 2. Пользовательский интерфейс модуля оптимизации инвестирования

a)

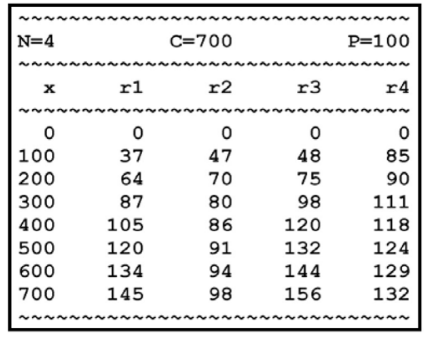

б)

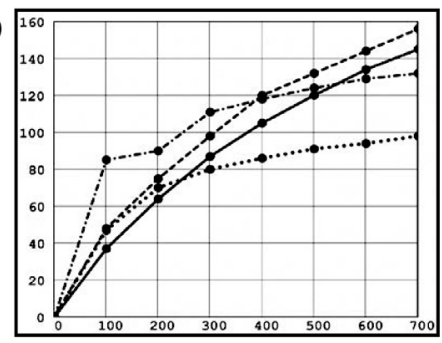

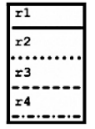

Рис. 3. Верификационный пример (начало) 


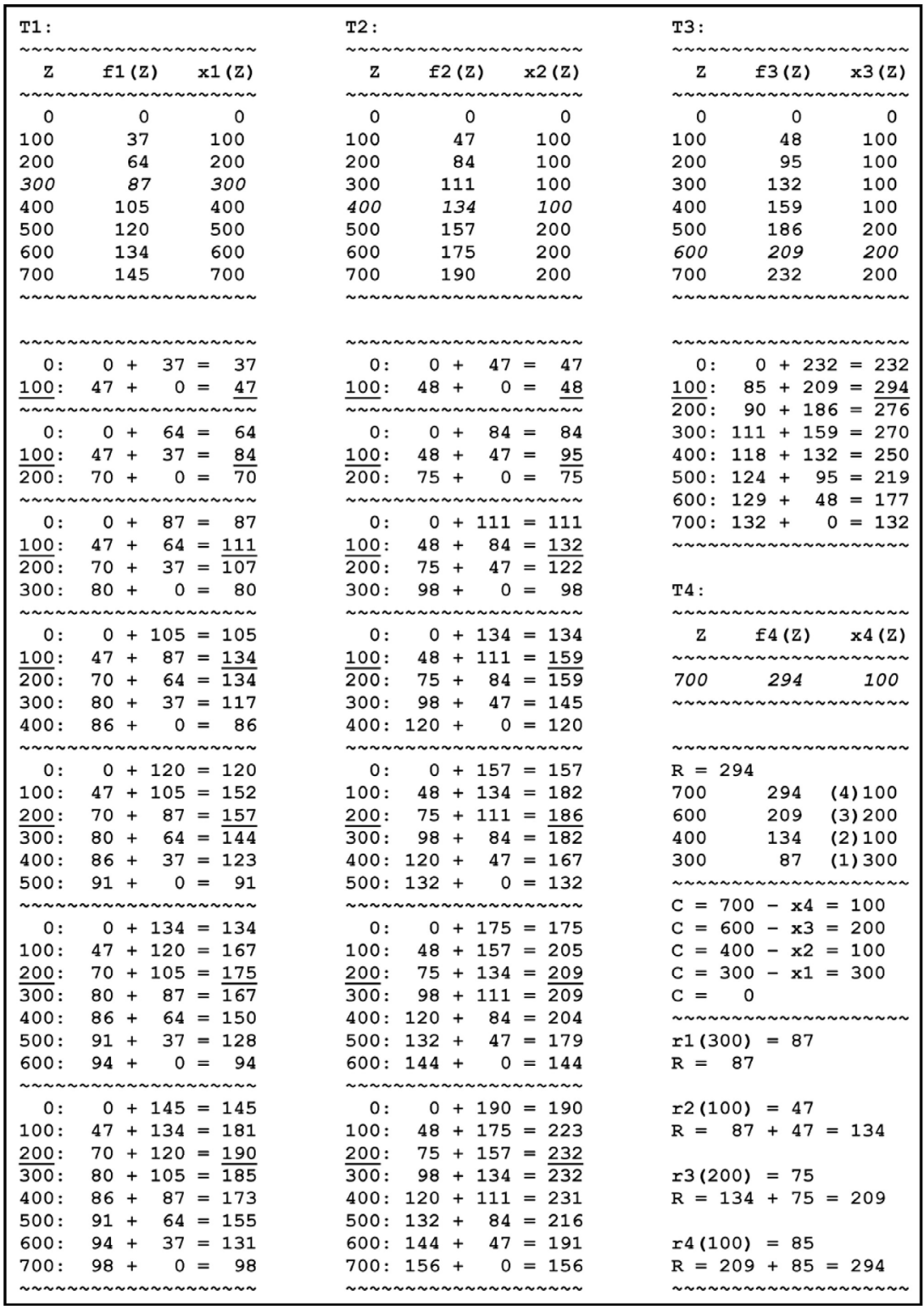

в)

Рис. 3. Продолжение 


\begin{tabular}{|c|c|c|c|c|c|c|c|c|c|}
\hline \multirow{2}{*}{\multicolumn{4}{|c|}{$\begin{array}{l}\text { T1: } \\
\sim \sim \sim \sim \sim \sim \sim \sim \sim \sim \sim \sim \sim \sim \sim \sim \sim\end{array}$}} & \multicolumn{4}{|l|}{ T2: } & \multicolumn{2}{|l|}{ т3: } \\
\hline & & & & \multicolumn{4}{|c|}{ 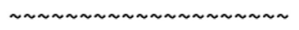 } & \multicolumn{2}{|c|}{ 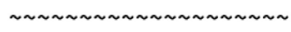 } \\
\hline $\mathrm{z}$ & \multicolumn{2}{|c|}{ f1 (z) } & $\mathrm{x} 1(\mathrm{z})$ & $\mathrm{z}$ & & $2(\mathrm{z})$ & $\mathrm{x} 2(\mathrm{z})$ & f3 (z) & x3(z) \\
\hline \multicolumn{4}{|c|}{ 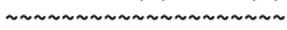 } & \multicolumn{4}{|c|}{ 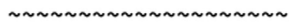 } & \multicolumn{2}{|c|}{ 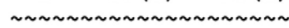 } \\
\hline 0 & & 0 & 0 & 0 & & 0 & 0 & 0 & 0 \\
\hline 100 & & 37 & 100 & 100 & & 47 & 100 & 100 & 100 \\
\hline 200 & & 64 & 200 & 200 & & 84 & 100 & 200 & 100 \\
\hline 300 & & 87 & 300 & 300 & & 111 & 100 & 300 & 100 \\
\hline 400 & & 105 & 400 & 400 & & 134 & 200 & 400 & 200 \\
\hline 500 & & 120 & 500 & 500 & & 157 & 200 & 500 & 200 \\
\hline 600 & & 134 & 600 & 600 & & 175 & 200 & 600 & 300 \\
\hline 700 & & 145 & 700 & 700 & & 190 & 200 & 700 & 300 \\
\hline \multicolumn{4}{|c|}{ 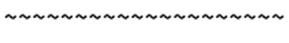 } & \multicolumn{4}{|c|}{ 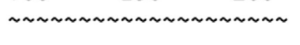 } & \multicolumn{2}{|c|}{ 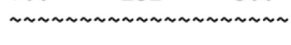 } \\
\hline \multicolumn{4}{|c|}{ 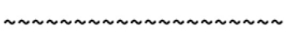 } & \multicolumn{4}{|c|}{ 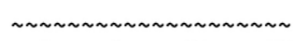 } & \multicolumn{2}{|c|}{ 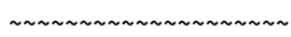 } \\
\hline $0:$ & 0 & +37 & $=37$ & $0:$ & 0 & +47 & $=47$ & $0+23$ & $32=232$ \\
\hline 100: & 47 & + & $=\underline{47}$ & 100: & 48 & + & $=\underline{48}$ & $85+20$ & $99=\underline{294}$ \\
\hline$\overline{\sim \sim \sim \sim a}$ & $\sim \sim \sim$ & 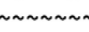 & $\sim \sim \sim \overline{\sim \sim}$ & $\overline{\sim \sim \sim \sim a}$ & น & 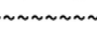 & 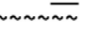 & $90+18$ & $36=\overline{276}$ \\
\hline $0:$ & 0 & +64 & $=64$ & $0:$ & 0 & +84 & $=84$ & $300: 111+15$ & $59=270$ \\
\hline 100: & 47 & +37 & $=84$ & 100: & 48 & +47 & $=\underline{95}$ & $400: 118+13$ & $32=250$ \\
\hline$\overline{200}:$ & 70 & +0 & $=\overline{70}$ & $\overline{200:}$ & 75 & + & $=\overline{75}$ & $500: 124+9$ & $95=219$ \\
\hline$\sim \sim \sim \sim \sim$ & $\sim \sim$ & $\sim \sim \sim \sim \sim \sim \sim$ & $\sim \sim \sim \sim \sim \sim$ & $\sim \sim \sim \sim \sim$ & $\sim \sim$ & $\sim \sim \sim \sim \sim \sim \sim$ & $\sim \sim \sim \sim \sim \sim$ & $600: 129+4$ & $48=177$ \\
\hline $0:$ & 0 & +87 & $=87$ & $0:$ & 0 & +111 & $=111$ & $700: 132+$ & $0=132$ \\
\hline 100: & 47 & +64 & $=111$ & 100: & 48 & +84 & $=132$ & $\sim \sim \sim \sim \sim \sim \sim \sim \sim \sim \sim \sim \sim$ & 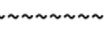 \\
\hline$\overline{200}$ : & 70 & $+\quad 37$ & $=\overline{107}$ & $\overline{200}$ : & 75 & +47 & $=\overline{122}$ & & \\
\hline $300:$ & 80 & + & $=80$ & $300:$ & 98 & + & $=98$ & $\mathrm{~T} 4:$ & \\
\hline$\sim \sim \sim \sim \sim$ & $\sim \sim \sim$ & 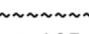 & $\sim \sim \sim \sim \sim \sim$ & $\sim \sim \sim \sim \sim$ & $\sim \sim$ & 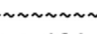 & 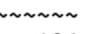 & $\sim \sim \sim \sim$ & \\
\hline $0:$ & 0 & +105 & $=105$ & $0:$ & 0 & +134 & $=134$ & $f 4(z)$ & $\mathrm{x} 4(\mathrm{z})$ \\
\hline 100: & 47 & +87 & $=134$ & 100: & 48 & +111 & $=159$ & 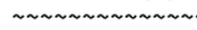 & $\sim \sim \sim \sim \sim \sim \sim \sim$ \\
\hline 200: & 70 & 64 & $=134$ & 200: & 75 & +84 & $=159$ & 700 & 100 \\
\hline$\overline{300}:$ & 80 & $+\quad 37$ & $=\overline{117}$ & $\overline{300}:$ & 98 & +47 & $=\overline{145}$ & 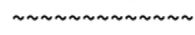 & $\sim \sim \sim \sim \sim \sim$ \\
\hline 400: & 86 & + & $=86$ & 400: & 120 & +0 & $=120$ & & \\
\hline$\sim \sim \sim \sim \sim$ & $\sim \sim \sim$ & 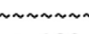 & 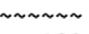 & $\sim \sim \sim \sim \sim$ & $\sim \sim \sim$ & 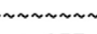 & 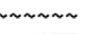 & & \\
\hline $0:$ & 0 & +120 & $=120$ & $0:$ & 0 & +157 & $=157$ & $R=294$ & \\
\hline 100: & 47 & +105 & $=152$ & 100: & 48 & +134 & $=182$ & 700 & (4) 100 \\
\hline 200: & 70 & $\begin{array}{r}+\quad 87 \\
\end{array}$ & $=157$ & 200: & 75 & +111 & $=186$ & 600 & (3) 300 \\
\hline$\overline{300}:$ & 80 & +64 & $=\overline{144}$ & $\overline{300}:$ & 98 & +84 & $=\overline{182}$ & 300 & (2) 100 \\
\hline 400: & 86 & 37 & $=123$ & 400: & 120 & +47 & $=167$ & 200 & (1) 200 \\
\hline 500: & 91 & + & $=91$ & 500: & 132 & + & $=132$ & 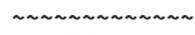 & 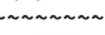 \\
\hline$\sim \sim \sim \sim \sim$ & $\sim \sim \sim$ & 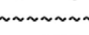 & $\sim \sim \sim \sim \sim \sim$ & $\sim \sim \sim \sim$ & $\sim \sim \sim$ & 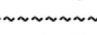 & 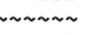 & $C=700-x 4$ & $=100$ \\
\hline $0:$ & 0 & +134 & $=134$ & $0:$ & 0 & +175 & $=175$ & $C=600-\times 3$ & $=300$ \\
\hline 100: & 47 & +120 & $=167$ & 100: & 48 & +157 & $=205$ & $C=300-x 2$ & $=100$ \\
\hline 200: & 70 & +105 & $=\underline{175}$ & $200:$ & 75 & +134 & $=209$ & $C=200-x 1$ & $=200$ \\
\hline $300:$ & 80 & +87 & $=167$ & $300:$ & 98 & +111 & $=209$ & $\mathrm{C}=0$ & \\
\hline 400: & 86 & +64 & $=150$ & $\overline{400}:$ & 120 & +84 & $=\overline{204}$ & 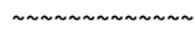 & 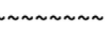 \\
\hline 500: & 91 & $+\quad 37$ & $=128$ & 500: & 132 & +47 & $=179$ & $r 1(200)=64$ & \\
\hline $600:$ & 94 & + & $=94$ & $600:$ & 144 & + & $=144$ & $R=64$ & \\
\hline$\sim \sim \sim \sim \sim$ & $\sim \sim \sim$ & 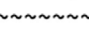 & $\sim \sim \sim \sim \sim \sim$ & $\sim \sim \sim \sim$ & $\sim \sim \sim \sim$ & $\sim \sim \sim \sim \sim \sim \sim$ & $\sim \sim \sim \sim \sim \sim$ & & \\
\hline 0: & 0 & +145 & $=145$ & $0:$ & 0 & +190 & $=190$ & $r 2(100)=47$ & \\
\hline $100:$ & 47 & +134 & $=181$ & 100: & 48 & +175 & $=223$ & $R=64+47$ & $=111$ \\
\hline 200: & 70 & +120 & $=190$ & 200: & 75 & +157 & $=232$ & & \\
\hline $300:$ & 80 & +105 & $=\overline{185}$ & $300:$ & 98 & +134 & $=232$ & $r 3(300)=98$ & \\
\hline 400: & 86 & +87 & $=173$ & $\overline{400}:$ & 120 & +111 & $=\overline{231}$ & $R=111+98$ & $=209$ \\
\hline 500: & 91 & +64 & $=155$ & 500: & 132 & +84 & $=216$ & & \\
\hline $600:$ & 94 & $+\quad 37$ & $=131$ & $600:$ & 144 & +47 & $=191$ & $r 4(100)=85$ & \\
\hline $700:$ & 98 & + & $=98$ & $700:$ & 156 & + & $=156$ & $R=209+85$ & $=294$ \\
\hline$\sim \sim \sim \sim \sim$ & 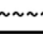 & $\sim$ & & $\sim \sim \sim$ & $\sim \sim \sim$ & $\sim \sim \sim \sim \sim \sim$ & $\sim \sim \sim \sim \sim \sim$ & $\sim \sim \sim \sim \sim \sim \sim \sim \sim \sim \sim \sim \sim$ & $\sim \sim$ \\
\hline
\end{tabular}

г)

Рис. 3. Окончание 

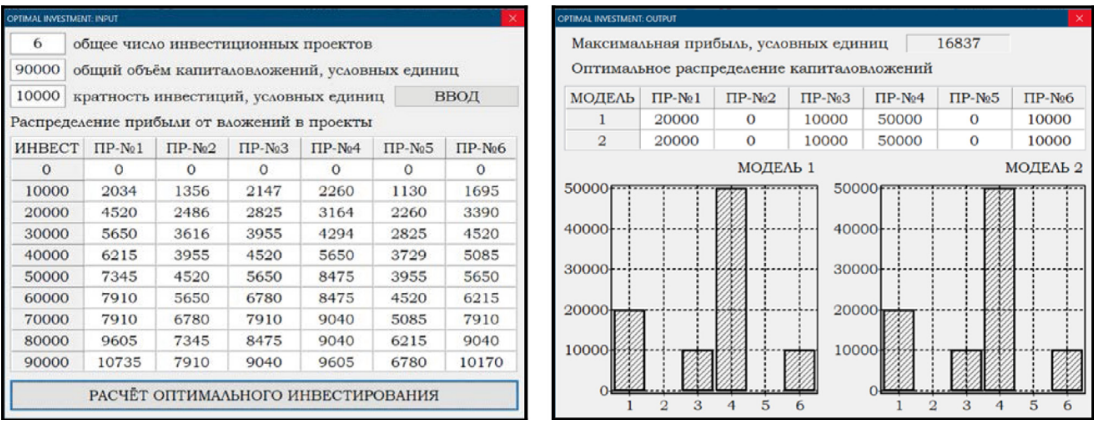

Рис. 4. Результат работы программы для совпадающих моделей инвестирования

Полученный по результатам проведенного исследования формализованный и высокодетализированный алгоритм динамического программирования обеспечивает и существенно облегчает генерацию соответствующего машинного кода прикладных программ оптимального инвестирования, а разработанное программное обеспечение позволяет: 1) оперативно определить оптимальное распределение капиталовложений, приносящее максимальную прибыль; 2) провести расчет как для одной, так и для нескольких моделей инвестирования с возможностью численного и графического сравнения и сопоставления; 3) представить численное обоснование принятого инвестиционного решения в формате верификационного примера с подробным протоколом расчета.

\section{Список литературы}

1. Архипкина А.И. Метод динамического программирования как инструмент поддержки принятия решения при планировании инвестиционного проекта // Наука и образование: новое время. 2018. № 3 (26). C. 248-257.

2. Баркалов С.А., Свиридова Т.А., Золотарев В.Н., Черненький А.В., Федосова С.П., Десятириков Ф.А. Актуализация распределения инвестиций методом динамического программирования // Международная конференция по мягким вычислениям и измерениям. 2018. T. 2. С. 351-354. 
3. Гафаров Е.Р. Графический метод динамического программирования с использованием кусочно-линейных функций Беллмана: Автореф. дис. ... д-ра физ.-мат. наук. Москва, 2016. 22 с.

4. Карасева Р.Б. Оптимальное распределение инвестиций по объектам вложения методами динамического программирования // Научнометодический электронный журнал Концепт. 2016. № 7. С. 62-67.

5. Корнеенко В.П., Данилюк А.Ю., Кутахов В.П., Филиппов П.Г. Динамическая модель управления инвестированием средств в неделимые проекты производства по критерию максимальной эффективности для отрасли // Экономика и предпринимательство. 2014. № 11-3 (52). C. 997-1001.

6. Кривошапова Г.А., Яркина А.А., Иценко М.Ю. Решение задачи инвестирования инновационных проектов с помощью метода динамического программирования // Теория и практика современной науки. 2020. № 1 (55). С. 456-464.

7. Москаленко В.В., Захарова Т.В., Криворука А.Н. Модели и процедуры формирования портфеля инвестиционных проектов на основе динамического подхода // Восточно-европейский журнал передовых технологий. 2014. Т. 3. № 3 (69). С. 29-32.

8. Сельвинский В.В., Мамаев В.О. Оптимальное распределение инвестиций при планировании производства // Вестник Амурского государственного университета. Серия: Естественные и экономические науки. 2020. № 89. С. 9-12.

9. Фролов С.В. Оптимальная инвестиционная политика предприятий с помощью метода динамического программирования // Форум молодых ученых. 2017. № 5 (9). С. 2183-2188.

10. Ходос Д.В., Шапорова В.Ю. Формирование оптимального графика инвестирования инновационного проекта промышленного предприятия на основе метода динамического программирования // Актуальные проблемы авиации и космонавтики. 2016. Т. 2. № 12. С. 451-453.

11. Hu Q., Szmerekovsky J. Project portfolio selection: a newsvendor approach // Decision sciences. 2017. Vol. 48. No. 1, pp. 176-199.

12. Khalifa H. A study on investment problem in chaos environment // Journal of Applied Research on Industrial Engineering. 2019. Vol. 6. No. 3, pp. 177-183. 
13. Lee H.S., Shin Y.H. A dynamic programming approach to subsistence consumption constraints on optimal consumption and portfolio // Journal of computational analysis and applications. 2017. Vol. 22. No. 1, pp. 79-99.

14. Shin Y.H., Koo J.L., Roh K.H. An optimal consumption and investment problem with quadratic utility and subsistence consumption constraints: a dynamic programming approach // Mathematical modelling and analysis. 2018. Vol. 23. No. 4, pp. 627-638.

15. Shin Y.H., Roh K.H. An optimal consumption and investment problem with stochastic hyperbolic discounting // Advances in difference equations. 2019. Vol. 1, p. 211.

\section{References}

1. Arkhipkina A.I. Metod dinamicheskogo programmirovaniya kak instrument podderzhki prinyatiya resheniya pri planirovanii investitsionnogo proekta [Dynamic programming as a tool to support decision-making for the investment project planning]. Nauka i obrazovanie: novoe vremya. 2018. No. 3 (26), pp. 248-257.

2. Barkalov S.A., Sviridova T.A., Zolotarev V.N., Chernen'kiy A.V., Fedosova S.P., Desyatirikov F.A. Aktualizatsiya raspredeleniya investitsiy metodom dinamicheskogo programmirovaniya [Updating the investment allocation method of dynamic programming]. Mezhdunarodnaya konferentsiya po myagkim vychisleniyam i izmereniyam. 2018. No. 2, pp. 351-354.

3. Gafarov E.R. Graficheskiy metod dinamicheskogo programmirovaniya s ispol'zovaniem kusochno-lineynykh funktsiy Bellmana [The graphical method of dynamic programming with piecewise linear Bellman functions]. Avtoref. dis. ... d-ra fiz.-mat. nauk. Moskva, 2016. 22 p.

4. Karaseva R.B. Optimal'noe raspredelenie investitsiy po ob"ektam vlozheniya metodami dinamicheskogo programmirovaniya [The optimal distribution of investments between deposits by method of dynamic programming]. Nauchno-metodicheskiy elektronnyy zhurnal Kontsept. 2016. No. 7, pp. 62-67.

5. Korneenko V.P., Danilyuk A.Yu., Kutakhov V.P., Filippov P.G. Dinamicheskaya model' upravleniya investirovaniem sredstv $\mathrm{v}$ nedelimye proekty proizvodstva po kriteriyu maksimal'noy effektivnosti dlya otrasli 
[Dynamic model of investing management in indivisible projects on the criterion of maximum efficiency for the industry]. Ekonomika i predprinimatel'stvo. 2014. No. 11-3 (52), pp. 997-1001.

6. Krivoshapova G.A., Yarkina A.A., Itsenko M.Yu. Reshenie zadachi investirovaniya innovatsionnykh proektov s pomoshch'yu metoda dinamicheskogo programmirovaniya [Solution of problem of investments in innovative projects with the use of dynamic programming]. Teoriya $i$ praktika sovremennoy nauki. 2020. No. 1 (55), pp. 456-464.

7. Moskalenko V.V., Zakharova T.V., Krivoruka A.N. Modeli i protsedury formirovaniya portfelya investitsionnykh proektov na osnove dinamicheskogo podkhoda [Models and procedures of investment projects potrfolio construction based on dynamic approach]. Vostochno-evropeyskiy zhurnal peredovykh tekhnologiy. 2014. Vol. 3. No. 3 (69), pp. 29-32.

8. Sel'vinskiy V.V., Mamaev V.O. Optimal'noe raspredelenie investitsiy pri planirovanii proizvodstva [Optimal allocation of investments in production planning]. Vestnik Amurskogo gosudarstvennogo universiteta. Seriya: Estestvennye i ekonomicheskie nauki. 2020. No. 89, pp. 9-12.

9. Frolov S.V. Optimal'naya investitsionnaya politika predpriyatiy s pomoshch'yu metoda dinamicheskogo programmirovaniya [The optimal policy of enterprise investments using the dynamic programming method]. Forum molodykh uchenykh. 2017. No. 5 (9), pp. 2183-2188.

10. Khodos D.V., Shaporova V.Yu. Formirovanie optimal'nogo grafika investirovaniya innovatsionnogo proekta promyshlennogo predpriyatiya na osnove metoda dinamicheskogo programmirovaniya [The formation of the optimal schedule of investments innovative project of the industrial enterprise on the basis of the method of dynamic programming]. Aktual'nye problemy aviatsii i kosmonavtiki. 2016. Vol. 2. No. 12, pp. 451-453.

11. Hu Q., Szmerekovsky J. Project portfolio selection: a newsvendor approach. Decision sciences. 2017. Vol. 48. No. 1, pp. 176-199.

12. Khalifa H. A study on investment problem in chaos environment. Journal of Applied Research on Industrial Engineering. 2019. Vol. 6. No. 3, pp. 177-183.

13. Lee H.S., Shin Y.H. A dynamic programming approach to subsistence consumption constraints on optimal consumption and portfolio. Journal of computational analysis and applications. 2017. Vol. 22. No. 1, pp. 79-99. 
14. Shin Y.H., Koo J.L., Roh K.H. An optimal consumption and investment problem with quadratic utility and subsistence consumption constraints: a dynamic programming approach. Mathematical modelling and analysis. 2018. Vol. 23. No. 4, pp. 627-638.

15. Shin Y.H., Roh K.H. An optimal consumption and investment problem with stochastic hyperbolic discounting. Advances in difference equations. 2019. Vol. 1, p. 211.

\section{ДАННЫЕ ОБ АВТОРЕ}

Тюльпинова Нина Владимировна, доцент кафедры «Технология машиностроения», кандидат технических наук, доцент Брянский государственный технический университет бульвар 50 лет Октября, 7, г. Брянск, 241035, Российская Федерачия ninatulpinova@mail.ru

\section{DATA ABOUT THE AUTHOR}

Tyulpinova Nina Vladimirovna, Associate Professor «Manufacturing engineering», Candidate of Engineering Sciences, Associate Professor

Bryansk State Technical University

7, 50 let Octyabrya Blvd, Bryansk, 241035, Russian Federation ninatulpinova@mail.ru

ORCID: 0000-0002-1485-7238 